Cómo citar este trabajo: Martín, Y. (2020). Analysis of hurricane-induced population spatial mobility through geospatial digital shadows derived from Twitter. Boletín de la Asociación de Geógrafos Españoles, 84, 2848, 1-35. https://doi.org/10.21138/bage.2848

\title{
Análisis de la movilidad espacial de la población asociada a huracanes \\ a partir de la sombra digital geoespacial derivada de Twitter
}

\author{
Analysis of hurricane-induced population spatial mobility \\ through geospatial digital shadows derived from Twitter
}

\author{
Yago Martín \\ ymart92@outlook.com
}

Departamento de Administración Pública

University of Central Florida (Estados Unidos)

\section{Resumen}

Múltiples investigadores creen que el estudio del comportamiento y movilidad espacial de la población ha alcanzado un cuello de botella debido a la rigidez de los métodos tradicionales de investigación en el campo y a la dificultad de acceso a información relevante y de confianza. La sombra digital geoespacial es una de las oportunidades más prometedoras para poder desarrollar y probar nuevas hipótesis en el estudio del comportamiento espacial, pero la aplicación de estos nuevos métodos todavía no ha sido suficientemente explorada en el campo de los riesgos y desastres. Este artículo recoge los últimos avances en este ámbito centrándose en la capacidad de la sombra digital geoespacial de redes sociales (Twitter) como un método innovador para el estudio del comportamiento espacial humano durante emergencias. Esta investigación rastrea las localizaciones de usuarios de Twitter durante el periodo pre-desastre para producir estimaciones 
del número de evacuados, y en los meses posteriores al desastre para estimaciones de desplazados y del impacto del evento en el turismo.

Palabras clave: comportamiento espacial; desastres; sombra digital espacial; Twitter; redes sociales.

\begin{abstract}
Multiple researchers believe spatial behavior has reached a bottleneck derived from the rigidity of traditional spatial behavior inquiry methods and the unavailability of trustworthy and relevant information. Digital geospatial trace data is seen as one of the most promising opportunities to develop and test new hypotheses on spatial behavior, but the application of these new methods has not been fully explored within the hazard/disaster discipline. This article summarizes the latest advancements in this area focusing on the suitability of geotagged social media (Twitter) as an innovative approach for human spatial behavior. This research tracks pre-event Twitter users' locations to produce estimates on the number of (pre-event) evacuees, and their post-disaster locations to estimate the number of displaced people, and the impact of a disaster on tourist visitations.
\end{abstract}

Key words: spatial behavior; disasters; digital spatial shadow; Twitter; social media.

\title{
1 Introducción
}

La movilidad espacial humana, definida como la capacidad del ser humano de cambiar de localización física en el espacio geográfico, ha experimentado una larga evolución a lo largo de la historia, siendo causa y consecuencia de numerosos cambios sociales (Manning, 2012).

cambio en la capacidad de movilidad del ser humano ha conllevado una transformación de sus relaciones desde una perspectiva local a una perspectiva global, extendiendo los límites espaciotemporales humanos hasta áreas difícilmente imaginables siglos atrás.

La revolución en las tecnologías del transporte ha propiciado esta rápida transformación en las últimas décadas. Durante gran parte de la historia humana, el hombre no dispuso más que de su fuerza física, de la fuerza animal, o del viento (barcos) para transportarse. Así, los viajes de larga distancia, cargados de peligros e incertidumbres, estaban reservados a los más privilegiados. Con la llegada prácticamente simultanea de los automóviles y la aviación, el transporte se democratizó entre la sociedad y la movilidad paso rápidamente de ser un privilegio de unos pocos a ser una realidad para muchos (Manning, 2012). 
Esta revolución en los transportes, unida la tecnología de la información actual, ha conllevado el desarrollo de un fenómeno conocido como la compresión espacio-temporal (Harvey, 1989). Esta teoría defiende que la era de postmodernidad en la que vivimos implica y resulta de una conexión física y virtual nutrida de la aceleración en el intercambio de bienes, personas, información e ideas, convirtiendo al tiempo en una mercancía más del capitalismo actual. Algunos autores han interpretado la movilidad moderna en términos de poder, dividiendo la sociedad entre aquellos que se pueden desplazar y aquellos que no (Bauman, 2000).

La compresión espacio-temporal, también denominada en la literatura en inglés como mundo que encoge (shrinking world), afecta también al comportamiento espacial humano antes, durante, y después de emergencias (Adey, 2016). Por ejemplo, decenas de miles de personas murieron en Portugal y España a consecuencia del tsunami originado por el famoso terremoto de Lisboa de 1755 (Solares \& Arroyo, 2004). Hoy en día, aunque estas mismas zonas siguen siendo vulnerables al mismo fenómeno y pese a que los sistemas de comunicación de tsunamis no están tan desarrollados como en otras regiones del mundo (Lima et al., 2010), la comunicación y la capacidad de evacuación es infinitamente superior a la del siglo XVIII. Si el mismo evento se repitiera, es previsible que miles de personas conseguirían escapar a tiempo antes de la llegada de las primeras olas. Esta compresión espacio-temporal ha supuesto por tanto una excepcional aceleración de la capacidad de respuesta en situaciones de vida o muerte, lo que ha contribuido al descenso en el número de muertes provocadas por riesgos que pueden preverse con suficiente antelación (e.g. huracanes) (Willoughby, 2012).

El estudio de la movilidad humana ha sido un tema central en la ciencia geográfica (Cresswell, 2011), pero también para muchas otras disciplinas (e.g., sociología, economía, ciencias políticas, historia, antropología). La geografía del comportamiento nace de la interacción de la geografía, la psicología, y la sociología, y se centra en el estudio de las decisiones espaciales (Estébanez Álvarez, 1990). Esta subdisciplina se desarrolló especialmente en los años 70 como reacción al enfoque cuantitativo que dominaba las ciencias espaciales en las décadas anteriores. La geografía del comportamiento reconoció la capacidad de las personas de tomar decisiones espaciales basadas en ciertas creencias, sentimientos, conocimientos, o emociones que determinan su cognición (Estébanez Álvarez, 1990). En este sentido, se entiende que el proceso de decisión espacial es mucho más complejo de lo que se había pensado hasta entonces y que diferentes individuos, grupos, y sociedades actúan de forma diferente ante los mismos estímulos. Uno de estos estímulos son los riesgos, ya sean naturales o antrópicos. Los primeros trabajos en esta línea emanan del influyente geógrafo americano Gilbert White (White, 1945), así como de sus 
discípulos académicos (Kates, 1962; Burton \& Hewitt, 1971), y continúan con aportaciones provenientes de la psicología, destacando el trabajo de Paul Slovic (Slovic, 1987).

La relocalización temporal es considerada como una de las formas más efectivas de reducir el peligro físico antes de la llegada de una amenaza (Bowser \& Cutter, 2015). El estudio de evacuaciones ha revelado que el riesgo y la incertidumbre, así como la capacidad de movilidad (también conocida como motilidad), son los mayores condicionantes en el proceso de toma de decisión acerca de evacuar o quedarse en la zona en riesgo. La incertidumbre se caracteriza por la ausencia de un conocimiento completo, mientras que el riesgo se asocia con la evaluación que hacen los sujetos de eventos inciertos. La interacción entre ambos provoca una toma de decisiones que puede alejarse de lo considerado lógico o racional (Golledge \& Stimson, 1997). La capacidad de movilidad es producto de factores como la disponibilidad de vehículo privado, la edad, o el estado socioeconómico, entre otros (Lindell et al., 2011). La literatura científica ha revelado que son muchos los factores con influencia en la valoración del riesgo y en la capacidad de evacuación. Por ejemplo, la localización (Zhang et al., 2004; Arlikatti et al., 2006), las características de la vivienda (Whitehead et al., 2000; Lindell et al., 2011), los avisos o alertas (Sorensen \& Mileti, 1988), factores demográficos (Gladwin \& Peacock, 1997; Dow \& Cutter, 1998), o la experiencia (Dow \& Cutter, 1998; Brommer \& Senkbeil, 2010).

La relocalización, de una duración variable que puede variar desde unas pocas semanas hasta años, o incluso ser permanente (migración), es otra respuesta espacial común después (postevento) de cambios ambientales producidos por cambios climáticos o por desastres puntuales (McLeman \& Gemenne, 2018). Los movimientos de desplazados o refugiados obedecen a un cambio de localización forzado y no previsto del área de residencia debido a los impactos de un desastre, mientras que la migración se considera más como un cambio permanente de lugar de residencia después una meditada decisión en base a las dificultades económicas y sociales en un área afectada (Adger et al., 2018). Sin embargo, esta distinción tiene muchos matices, y un desplazado temporal puede terminar siendo un migrante (Black et al., 2013; Adger et al., 2018). Estos fenómenos se han estudiado desde diferentes perspectivas, de forma especial después de la gran diáspora producida por el Huracán Katrina en 2005. Distintos investigadores se han centrado en la distribución espacial de los evacuados, desplazados, y migrantes, así como en su toma de decisiones (Fussell et al., 2010; Groen \& Polivka, 2010).

Tradicionalmente, el estudio de los procesos de evacuación o de relocalización post-evento se ha basado en el análisis de estadísticas oficiales como censos (con problemas de agregación y baja 
periodicidad), o trabajos de detalle mediante encuestas. Algunos autores han destacado la gran dificultad en el estudio de los movimientos humanos dada la ausencia de datos de confianza, algo todavía más prevalente durante emergencias o después de desastres (Laczko, 2015; Rango \& Vespe, 2017). Finch et al. (2010) destacan la gran ausencia de datos acerca de residentes huyendo de zonas afectadas por desastres, desconociendo en muchos casos su destino y si estos desplazamientos son temporales o permanentes. En este sentido, aunque centrado en evacuaciones, Baker (2009) hacía un llamamiento para encontrar innovaciones en la colección de datos:

"necesitamos emplear nuevos métodos o combinaciones de métodos en la colección de datos... Si los combinamos con los métodos tradicionales, podrían ayudar a fortalecer y expandir los conocimientos actuales sobre el comportamiento durante evacuaciones, evaluando al mismo tiempo su credibilidad al confrontarlos con medidas cuya fiabilidad es conocida...".

Los obstáculos y dificultades para seguir la trayectoria de los evacuados, desplazados, o migrantes en un "mundo que encoge" son todavía más grandes, ya que en pocas horas las personas afectadas por un desastre pueden encontrarse a miles de kilómetros del lugar, traspasando incluso las fronteras nacionales. La aceleración en la movilidad generada por emergencias provoca por tanto la dificultad de los métodos tradicionales, con frecuencia demasiado rígidos, de conseguir dar respuesta a un proceso tan dinámico.

La revolución digital que ha favorecido la compresión espacio-temporal, especialmente a partir de la masificación de internet y del uso de ordenadores y teléfonos móviles, conlleva también la aparición de oportunidades para el estudio de los movimientos humanos. En esta nueva era digital, también llamada Era de la Información, la producción de datos digitales producto del uso de dispositivos electrónicos y plataformas web es inmensa. Este enorme volumen de datos se conoce como "Big Data", y ha sido caracterizados como las 3+1 "V": Volumen, Velocidad, Variedad, y más recientemente, Valor (Laczko, 2015). Entre las muchas clases y formatos de Big Data existentes, el Big Dała geoespacial es de gran interés para la Geografía. De forma más específica, la sombra (o huella) digital geoespacial, también conocida como datos de ciudadanos-sensores pasivos, es particularmente relevante para el estudio del comportamiento espacial humano. El uso y aprovechamiento de esta sombra digital en situaciones de no emergencia ha sido amplio en los últimos años (e.g. Wesolowski et al., 2012; Amini et al., 2014; Çolak et al., 2016). Sin embargo, 
su aplicación en estudios de movilidad o comportamiento espacial en situaciones de emergencia o desastres ha sido bastante más reducida (e.g. Gething \& Tatem, 2011; Yabe et al., 2019).

Este articulo tiene como objetivo la síntesis de las nuevas posibilidades que ofrece el estudio de las sombras digitales geoespaciales en el estudio de la movilidad producida por desastres. El artículo se centra particularmente en la introducción de las nuevas fuentes de datos para el estudio del comportamiento espacial humano, prestando especial atención en su aplicación en casos relacionados con huracanes. De este modo, este documento plantea una propuesta metodológica basada en la sombra digital geoespacial de la red social Twitter para resolver tres cuestiones fundamentales aplicadas a dos casos de estudio: 1) calcular la tasa de evacuación de distintos grupos ante un huracán, 2) estimar el porcentaje de desplazados después de un huracán, y 3) estimar la afección en la llegada de visitantes a una zona severamente afectada por un huracán.

\section{El estudio del comportamiento espacial humano durante emergencias}

\subsection{Enfoques tradicionales}

Los métodos tradicionales de estudio de la movilidad de la población en situaciones de peligro son las encuestas (cuestionarios) y las entrevistas. Los cuestionarios han sido el método preferido por los investigadores para conocer el comportamiento de la población en evacuaciones provocadas por huracanes (e.g. Siebeneck \& Cova, 2012), tornados (Durage et al., 2014), terremotos, (Tamima \& Chouinard, 2016), tsunamis (San Carlos Arce et al., 2017) o riesgos no naturales como los accidentes nucleares (Zeigler et al., 1981) o de productos peligrosos (Mitchell et al., 2007).

Algunas características de las encuestas las hacen atractivas para obtener un buen grado de conocimiento acerca de los factores condicionantes que determinan las decisiones espaciales y la planificación de evacuaciones. Por ejemplo, el investigador posee el control sobre la muestra y las preguntas a realizar. Esta flexibilidad es la mayor ventaja de este método. Para el estudio de evacuaciones, las encuestas ofrecen relativa alta resolución espacio-temporal, ya que el encuestado es, generalmente, capaz de recordar sus movimientos y el momento de estos, aunque algunos autores cuestionan la validez debido al llamado sesgo de recuerdo (Shareck et al., 2013). Otra de las fortalezas de este método es la riqueza de datos que se tienen del encuestado y de su motivación para evacuar o permanecer en el área de riesgo (Baker, 2009). Por otra parte, las encuestas presentan limites que restringen su aplicación como método de estudio único y exhaustivo. Principalmente, los investigadores ponen en duda la representatividad de la muestra 
(Arlikatti et al., 2006). En las últimas décadas, la tasa de respuesta a encuestas y cuestionarios ha descendido (Johnson \& Wislar, 2012) y las muestras sufren de claros sesgos, ya que los encuestados suelen ser mayores que la media de edad de la población general y su distribución racial no suele seguir la distribución racial de la sociedad (Bowser, 2013). Además, no es desdeñable el alto grado de recursos temporales, humanos, y económicos necesarios en la realización de encuestas (Baker, 2009). Por último, las encuestas se han mostrado ineficaces para alcanzar a poblaciones flotantes o residentes temporales (e.g. turistas o trabajadores estacionales), no reflejando por tanto el comportamiento del conjunto de la población en riesgo (Smith \& McCarty, 2009).

Otro método frecuentemente utilizado en el análisis del comportamiento espacial durante evacuaciones son los sensores de tráfico instalados por los respectivos departamentos de tráfico. Una densa red de contadores ofrece una estimación precisa del número de vehículos que se encuentran en las carreteras en cada momento (tiempo real), lo que es una información muy relevante para las autoridades que gestionan la evacuación (Wolshon \& Pande, 2016). La accesibilidad de los datos y su bajo coste son algunas de las ventajas más consideradas de este método (Ballard et al., 2008; Archibald \& McNeil, 2012). Los inconvenientes más citados son la total ausencia de información acerca de los ocupantes de los vehículos. Así, el investigador es completamente ajeno al número de personas por vehículo además de a las características sociodemográficas de estas y a los motivos de su desplazamiento (Archibald and McNeil, 2012).

\subsection{Datos de sensores ciudadanos}

Muchos investigadores han comenzado a utilizar métodos alternativos para obtener información acerca del comportamiento espacial de la población en condiciones normales (e.g. Chaintreau et al., 2007; González et al., 2008; Jurdak et al., 2015), pero todavía existe una escasez de estudios bajo circunstancias de emergencia, donde el comportamiento espacial habitual puede no ser el mismo (Wang and Taylor, 2014). Con la llegada y la popularización de las tecnologías de geoposicionamiento incorporadas a dispositivos electrónicos y la expansión masiva del mundo digital, el individuo deja trazas de información (sombras de datos) que pueden ser aprovechadas en proyectos de investigación, entre otros usos. Estos datos, donde el individuo se convierte en un sensor, o en el portador del mismo, se conocen como datos de sensores ciudadanos o sensores humanos (citizen-sensor data or human-sensor data) (Goodchild, 2007). Estos sensores ciudadanos se pueden dividir en sensores ciudadanos activos y sensores ciudadanos pasivos. La diferencia fundamental entre ellos se determina por la conciencia del individuo sobre el uso final de los datos 
generados por el sensor (Birenboim \& Shoval, 2016). De este modo, los sensores ciudadanos activos son conscientes del uso final de los datos y participan voluntariamente, mientras que los ciudadanos sensores pasivos desconocen el uso final de los datos generados, o desconocen incluso que los están generando.

\section{a) Sensores ciudadanos activos}

Los datos de sensores ciudadanos activos en estudios de comportamiento espacial se ciñen al monitoreo de los participantes mediante Sistemas Globales de Navegación por Satélite (e.g. Global Positioning System, GPS) y otros sensores como acelerómetros. Este tipo de datos es común en disciplinas como Salud Pública. Por ejemplo, Vazquez-Prokopec et al. (2009) investigaron la posibilidad de conocer y cuantificar los movimientos de la población y su relación con la transmisión del virus del dengue en entornos urbanos tropicales mediante la utilización de dispositivos GPS. Otros muchos estudios han seguido este camino y han dotado de sensores GPS a distintas poblaciones para relacionar movilidad y transmisión de enfermedades infecciosas (Stanton et al., 2017; Falcón-Lezama et al., 2017). Otras disciplinas que han aprovechado estas tecnologías han sido el turismo (Shoval et al., 2011) o los estudios de transporte (Wang et al., 2015).

Muchos han visto en los sensores ciudadanos activos la posibilidad de superar los resultados obtenidos por los enfoques tradiciones como las encuestas (Elgethun et al., 2007). Alguna de las ventajas citadas son la alta precisión espacio-temporal ofrecida por los dispositivos GPS, lo que permite la reconstrucción y cuantificación del movimiento humano; lo asequible de los dispositivos, con frecuencia ya insertados en los teléfonos inteligentes y otros aparatos electrónicos; y la posibilidad de trabajar en entornos remotos (Paz-Soldan et al., 2014; Dueker et al., 2014). Por otra parte, también se han citado límites y desventajas de estas tecnologías, en especial en su aplicación a la investigación de movimientos poblaciones asociados a riesgos. Una de las grandes desventajas en este sentido es la ausencia de contexto sobre los motivos del desplazamiento, pero también la incapacidad de tener información en espacios cerrados. Otros autores han mencionado barreras culturales y bajos tamaños muestrales. Por último, pero no por ello menos importante, el hecho de que los participantes en el estudio conocen que su comportamiento espacial está siendo monitoreado introduce un marcado sesgo en los datos, ya que los individuos pueden cambiar sus decisiones, voluntaria o involuntariamente. 


\section{b) Sensores ciudadanos pasivos}

Los datos de sensores ciudadanos pasivos se basan en la simbra digital que los individuos generan en su actividad digital diaria. Las fuentes de sensores ciudadanos pasivos más utilizadas para el estudio del comportamiento espacial humano son los registros de teléfonos móviles, las tarjetas inteligentes, las redes Wi-Fi y Bluetooth, y las redes sociales. Las necesidades del estudio de comportamiento espacial son distintos en función de las emergencias (riesgos), por lo que es difícil crear un panorama general de las ventajas y debilidades de cada fuente de datos. Por ejemplo, lo que podría considerarse como una baja resolución espacial para el estudio del comportamiento espacial durante un ataque terrorista podría ser más que suficiente para estudiar una evacuación a gran escala durante un huracán.

Los registros de teléfonos móviles son una fuente de información espacial muy rica que provee de muestras representativas (prácticamente todos los individuos, sin importar condición, poseen un teléfono móvil) y una buena resolución tanto espacial como temporal (González et al., 2008; Birenboim \& Shoval, 2016). Las características de estos datos han atraído la atención de numerosas disciplinas como sociología (Amini et al., 2014), salud pública (Wesolowski et al., 2012), geografía (Phithakkitnukoon et al., 2012) o estudios urbanos (Bajardi et al., 2015). El uso de estos datos en situaciones de riesgo ha sido muy limitado. Solo epidemiólogos centrados en el estudio de riesgos biológicos han recogido y analizado de forma sistemática estos datos para inferir los movimientos humanos (Wesolowski et al., 2012; Vogel et al., 2015). En algunos casos, los datos móviles también han sido utilizados para comprender los procesos migratorios post-desastre, como en el caso de un terremoto (Bengtsson et al., 2011) o un huracán (Echenique \& Melgar, 2018). La accesibilidad a los datos (almacenados y protegidos por empresas privadas) y la ausencia de información sobre los individuos (normalmente restringida por razones de privacidad) son la mayor limitación de este tipo de datos (Widhalm et al., 2015).

Los datos de tarjetas inteligentes se remiten a la sombra digital producida por el uso de cualquier tarjeta con circuitos integrados, lo que es muy común en el mundo financiero, de las comunicaciones, identificaciones personales, transporte público, salud etc. Estudios de movilidad han aprovechado estos datos para explorar, por ejemplo, el comportamiento espacial de turistas extranjeros (Sobolevsky et al., 2014) o el dinamismo de espacios de actividad urbanos (Gong et al., 2017). La sombra digital de estas tarjetas inteligentes produce normalmente una muy alta resolución espacial (aunque sujeta al lugar del instrumento receptor) y una resolución temporal muy variable y dependiente del tipo de tarjeta (Zhong et al., 2015; Gong et al., 2017). La 
representatividad de las muestras depende del tipo de dato analizado, pero la gran penetración de estas tarjetas en la sociedad permite la obtención de muestreos representativos. La accesibilidad a los datos y la pobreza semántica de la información son de nuevo la mayor barrera en su utilización.

Las redes inalámbricas como Wi-Fi and Bluetooth tienen un enorme potencial de aplicación en la investigación del comportamiento espacial (Perttunen et al., 2014). Una barrera particular de estos datos es su reducida cobertura espacial, ya que la geolocalización de los individuos solo es posible dentro del alcance de la señal de los dispositivos (Kontokosta \& Johnson, 2017). A esto, hay que añadir la dificultad de acceder a los datos, la ausencia de información del portador del dispositivo (lo que complica conocer la representatividad de la muestra), y la privacidad y confidencialidad (Kontokosta \& Johnson, 2017). En el lado positivo de la balanza, a la capacidad de posicionamiento en tiempo real se une la capacidad de localizar dispositivos (y por tanto individuos) en entornos cerrados, lo que hace que esta tecnología tenga aplicaciones muy interesantes en la búsqueda de cuerpos enterrados en colapsos de edificios después de terremotos o avalanchas o para conocer el comportamiento espacial en ataques terroristas (Cooper et al., 2016; Moon et al., 2016).

Por último, la llegada de las redes sociales ha supuesto una revolución en el ámbito de la investigación social, con aplicaciones en numerosas disciplinas (e.g. Widener \& Li, 2014; Gerber, 2014; Li et al., 2018). Twitter se ha convertido en la mayor fuente de datos de redes sociales debido a su política de acceso abierto al 1\% de su contenido total (Burton et al., 2012), mientras que otras plataformas como Facebook permanecen virtualmente inexploradas por su estricta política de privacidad. La aplicación de datos de Twitter ha sido profusamente aprovechada en el campo de la gestión y comunicación de emergencias entre otros ámbitos (e.g. Ukkusuri et al., 2014; Spence et al., 2016). Sin embargo, la aplicación de la sombra digital geoespacial de redes sociales no ha sido tan explorada para el estudio del comportamiento espacial en emergencias. En este sentido, Wang and Taylor (2014, 2016) identificaron distintos patrones espacio-temporales de movilidad en distintos desastres. Martín et al. (2017) introdujeron un método para estimar la obediencia de una evacuación obligatoria utilizando la sombra digital geoespacial de Twitter. Este método ha sido replicado más recientemente en otros eventos como el Huracán Sandy (Kumar and Ukkusuri, 2018). Estos estudios han revelado las ventajas que este tipo de datos ofrecen: son datos cada vez más comunes en la sociedad actual y en particular entre los segmentos más jóvenes de la población; existe capacidad de procesado y análisis en tiempo real; y son datos simples y de libre acceso, siempre condicionado por la capacidad computacional y las habilidades de programación 
del investigador. Las debilidades más notorias de la sombra digital geoespacial proveniente de las redes sociales incluyen la baja resolución espacial, ya que solo una pequeña fracción de los tweets está georreferenciada con las coordenadas exactas, la baja periodicidad o resolución temporal, lo que Andrienko et al. (2012) definieron como datos dispersos y episódicos, y la representatividad de los datos (Martín et al., 2017).

\section{Metodología: Twitter como fuente de datos espaciales}

\section{1 Áreas de estudio y contexto de los desastres}

Las dos áreas de estudio de esta investigación son los condados costeros de Carolina del Sur (Estados Unidos) durante la evacuación provocada por el Huracán Matthew de octubre de 2016 y la isla de Puerto Rico durante y después del Huracán María en septiembre de 2017. La Tabla 1 recoge las áreas de estudio para cada pregunta de investigación, así como la escala espaciotemporal estudiada.

\section{Tabla 1. Áreas de estudio y escalas espacio-temporales}

$$
\text { Área Escala espacial Escala temporal }
$$

\begin{tabular}{llll}
\hline Evacuación & Condado & Día \\
Hurricane Matthew & Puerto Rico & Región & Día \\
$\begin{array}{l}\text { Migración post-desastre } \\
\text { Hurricane María }\end{array}$ & & \\
$\begin{array}{l}\text { Visitantes post-desastre } \\
\text { Hurricane Maria }\end{array}$ & Puerto Rico & Región & Semana \\
\hline
\end{tabular}

Fuente: elaboración propia

El Huracán Matthew fue el huracán más potente del Atlántico en la temporada 2016, siendo el primer huracán categoría 5 en esta cuenca desde 2007 (Stewart, 2017). Matthew se formó el 28 de septiembre de 2016, alcanzando categoría 5 tres días después tras un proceso de rápida intensificación (Figura 1). La tormenta se extratropicalizó frente a las costas de Carolina del Norte el 9 de octubre. Provocó un total de 585 muertes directas: 546 en Haití, 34 en los Estados Unidos, 4 en la República Dominicana, y 1 en San Vicente y las Granadinas (Stewart, 2017). Dada la trayectoria prevista del huracán, las autoridades ordenaron la evacuación de los residentes de las zonas costeras estadounidenses (desde Florida hasta Carolina del Norte).

María se convirtió en tormenta tropical al este de las Antillas Menores el 16 de septiembre. Después de un proceso de rápida intensificación alcanzó categoría 5 el 18 de septiembre, 
impactando Dominica con consecuencias catastróficas. Después de golpear Dominica, María se reforzó antes de cruzar Puerto Rico como un intenso huracán categoría 4 (Figura 1). Se estima que más de 4.600 personas perdieron la vida en Puerto Rico a causa de María tras desatarse una crisis humanitaria en la isla (Kishore et al., 2018).

Figura 1. Recorrido de los Huracanes Matthew (2016) y María (2017)

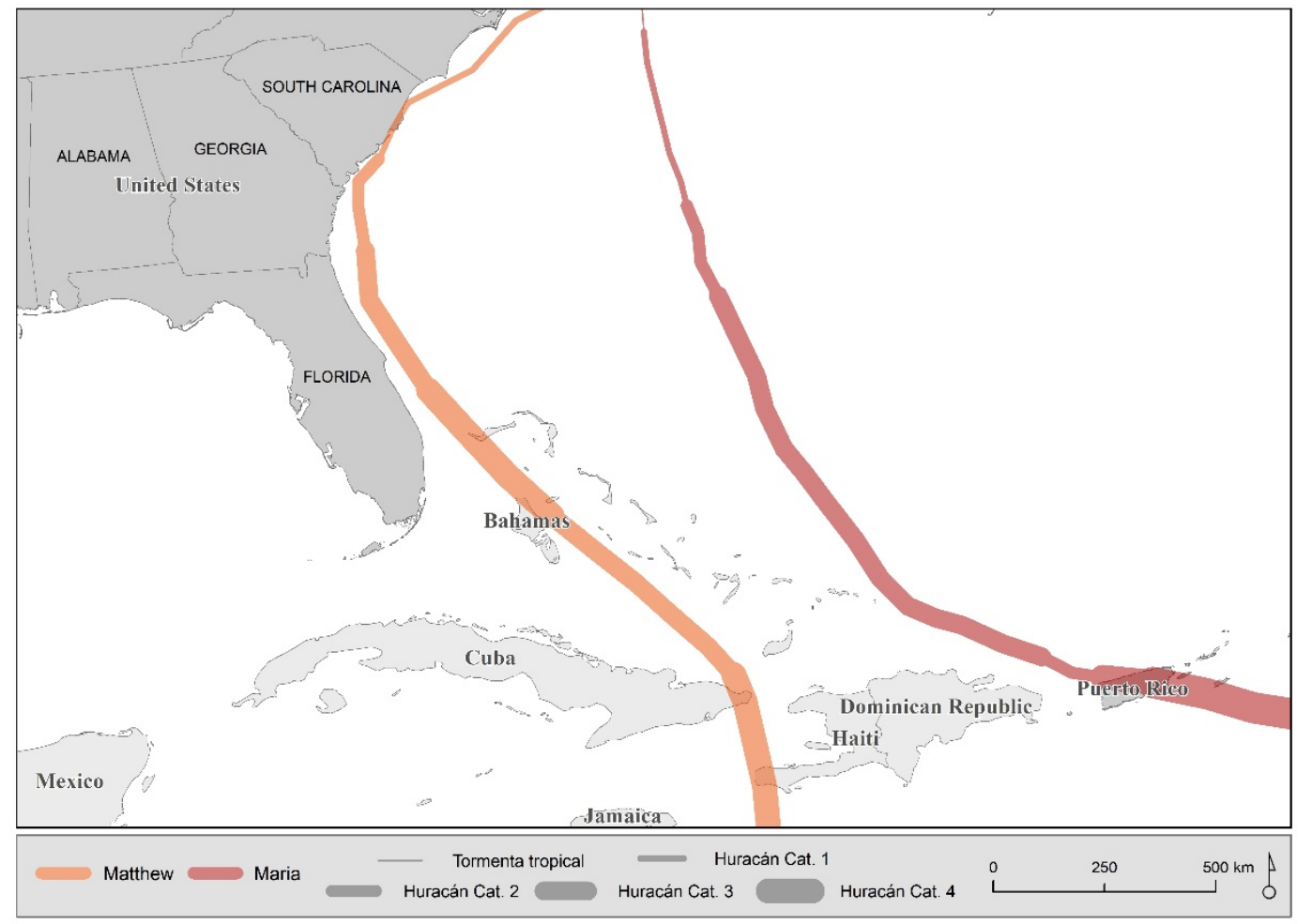

Fuente: elaboración propia a partir de NHC (2018)

\subsection{Adquisición de los datos}

La fuente de datos para estudiar el comportamiento espacial de las poblaciones afectadas proviene de la red social Twitter. La Figura 2 sintetiza el flujo de trabajo seguido para obtener estos datos. Desde julio de 2015, el departamento de Geografía de la Universidad de Carolina del Sur recoge y almacena de forma sistemática todos los tweets que la plataforma Twitter pone a disposición de la comunidad investigadora a través de su interfaz para programadores de aplicaciones (API). Los tweets se almacenan y gestionan en un entorno Hadoop (http://hadoop.apache.org) creando un repositorio del que se extraerán las diferentes bases de datos para los análisis (dependiendo de las necesidades). La Tabla 2 recoge las bases de datos inicialmente extraídas del repositorio principal. 
Figura 2. Flujo de trabajo para la obtención de datos

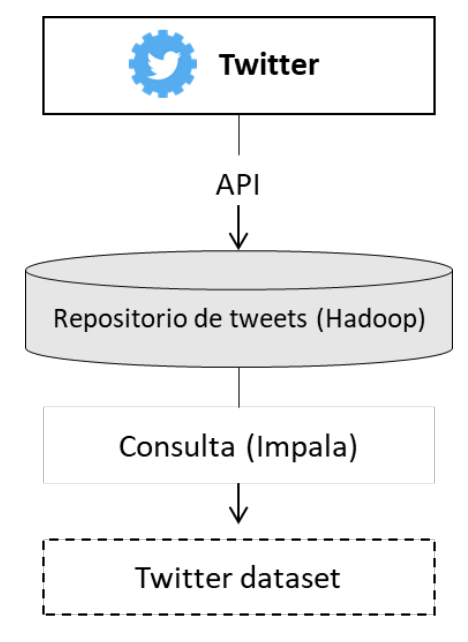

Fuente: elaboración propia

Tabla 2. Bases de datos extraídas inicialmente del repositorio

\begin{tabular}{llll} 
Nombre del dataset & Región & Tiempo & $\begin{array}{l}\text { Precisión } \\
\text { (georreferenciación del tweet) }\end{array}$ \\
\hline Pre-evacuación Matthew & $\begin{array}{l}\text { Condados costeros } \\
\text { de Carolina del Sur }\end{array}$ & $02 / 10-04 / 10,2016$ & Ciudad, Par de coordenadas \\
Post-evacuación Matthew & EEUU & $07 / 10-09 / 10,2016$ & Ciudad, Par de coordenadas \\
Pre-María & Puerto Rico & $01 / 09 / 2016-31 / 08 / 2017$ & Ciudad, Par de coordenadas \\
Post-María & Global & $01 / 09 / 2017-31 / 08 / 2018$ & Ciudad, Par de coordenadas \\
\hline
\end{tabular}

Fuente: elaboración propia

\subsection{Procesado y análisis de los datos}

a) Estimación del cumplimiento de evacuación

El método se basa en que cada usuario de Twitter posee un número identificativo único que permite analizar las localizaciones de sus tweets incluso aunque cambien de nombre en la red social. De este modo, el objetivo del primer conjunto de datos (Pre-evacuación Matthew) es identificar el mayor número de usuarios activos (distintas ID) en el área de estudio (condados costeros de Carolina del Sur) antes de la orden de evacuación. El periodo recogido abarca desde el 2 de octubre al 4 de octubre (día de la orden de evacuación) y la precisión espacial se define en ciudades y puntos (coordenadas exactas) para poder obtener agregados a nivel condado. El segundo conjunto de datos (Postevacuación Matthew) tiene como objetivo determinar la localización en los Estados Unidos de los usuarios activos durante el periodo de pre-evacuación en los condados costeros. El periodo de post-evacuación se determina entre las 6 de la tarde del 7 de 
octubre, donde se entiende que la evacuación ha concluido teniendo en consideración la noche y las condiciones de viaje (primeras bandas de lluvia de Matthew), y las 10 de la mañana del 9 de octubre considerando la noche y las condiciones de lluvia y posibles inundaciones de las horas previas (Dow \& Cutter, 2002). Los usuarios activos en ambos periodos (1384 usuarios) se someten a un análisis de la localización de sus tweets durante el año previo, pudiendo así conocer si son residentes de la zona de estudio o son visitantes temporales. Martín et al. (2017) y Martín et al. (2019) contienen información más detallada acerca del proceso seguido.

b) Estimación del porcentaje de desplazados / migrantes post-desastre

En el análisis de la respuesta espacial de los residentes de Puerto Rico después del Huracán María se analizó la muestra Pre-María (1 de septiembre, 2016 - 31 de agosto, 2017) con objeto de identificar usuarios de Twitter locales que estaban residiendo en la isla antes del huracán. Una vez obtenidos los 32099 usuarios locales se extrajeron los tweets de estos usuarios en todo el mundo durante el periodo post-María (1 de septiembre, 2017 - 31 de agosto, 2018). Después de un proceso de filtrado de cuentas con comportamiento de Twitter sospechoso (por ejemplo, cuentas multi-usuario con tweets en lugares distantes al mismo tiempo) y cuentas de Twitter no humanas (fuentes como Tweetbot for IS o TweetMyJOBS) y después de excluir usuarios que no publicaron al menos un tweet al mes durante el periodo post-María, el tamaño muestral final se compone de 1231 usuarios. Posteriormente, se analizaron los tweets de los 1231 usuarios de forma individual para conseguir información sobre sus movimientos post-desastre: estimación del porcentaje de desplazados, destinos, y el momento de la salida y del retorno.

\section{c) Estimación del número de visitantes post-desastre}

En el estudio del número de visitantes a Puerto Rico en el periodo post-desastre se compara la cantidad de usuarios de Twitter no residentes activos en la isla durante el periodo post-María con los valores pre-desastre (año anterior). Para distinguir los usuarios residentes de los usuarios no residentes se sigue un proceso similar a los casos anteriores (estudio de las localizaciones de los tweets de cada usuario en el año anterior al periodo analizado). Para analizar diferencias entre distintas zonas de la isla, pues los daños de María y las actividades económicas varían notablemente en la isla, Puerto Rico se dividió en 5 regiones (central, norte, oeste, sur y este) (Figura 3) y los datos semanales se agregaron en función de estas. 
Figura 3. División de Puerto Rico en cinco regiones, vientos estimados del huracán y marejada ciclónica

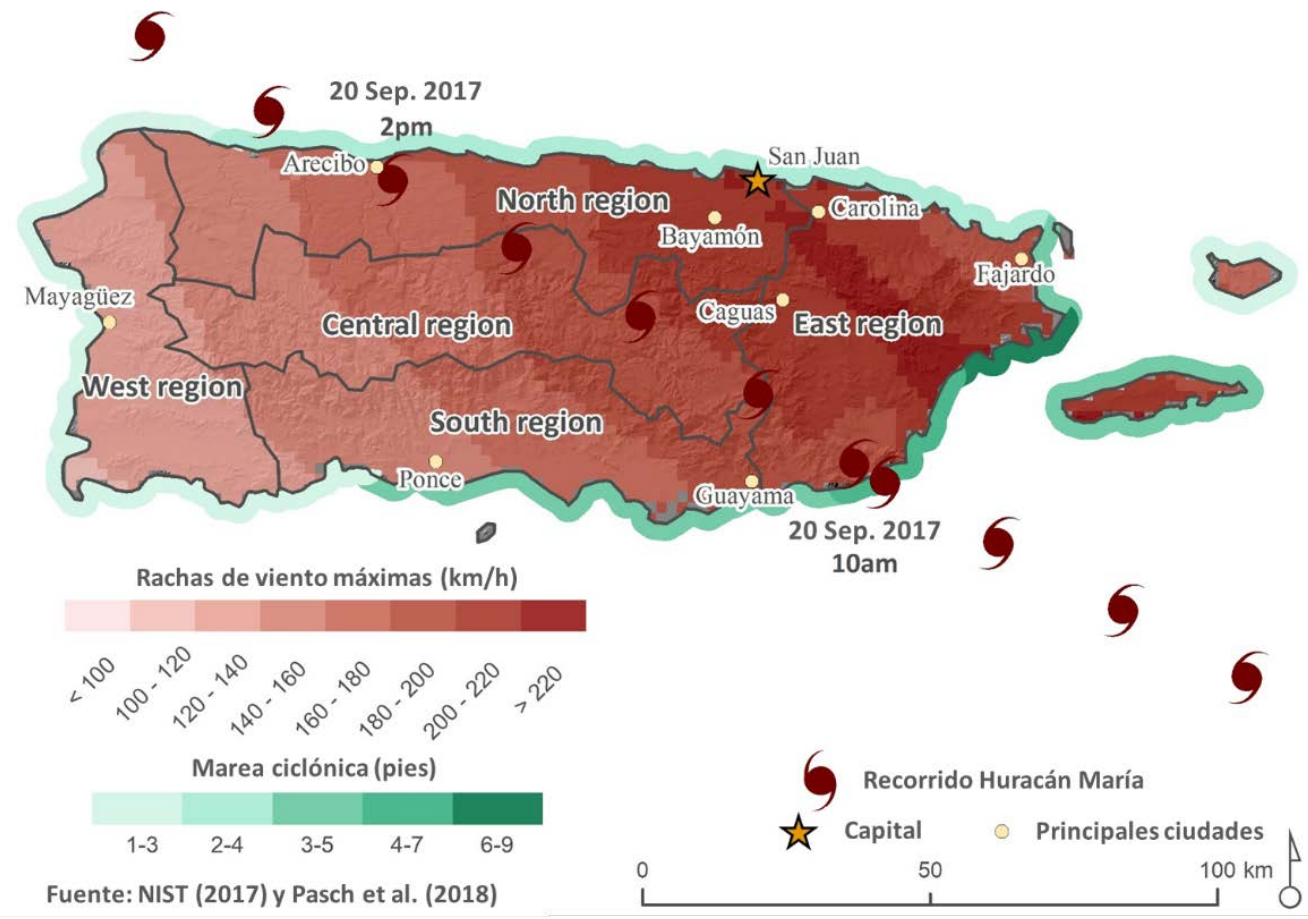

Fuente: elaboración propia a partir de NIST (2017 y Pasch et al. (2018)

\section{Resultados y discusión}

\subsection{Estimación del cumplimiento de evacuación}

La Tabla 3 muestra el número de usuarios evacuados, los porcentajes respectivos, y el número total de evacuados estimados en relación con el total de la población. La zona de estudio se divide en 3 conglomerados (sur, centro, y norte) siguiendo el estudio del comportamiento de evacuación de Carolina del Sur de 2011 (Cutter et al., 2011). Para el conjunto del área de estudio, el 54 \% (747 de 1384) de los usuarios locales evacuaron, una tasa que concuerda con estudios previos sobre evacuaciones hipotéticas (Cutter et al., 2011). En otro huracán de similar categoría, Floyd en 1999, Dow and Cutter (2000) observaron una tasa de evacuación del 65\%, lo que no dista mucho de la tasa observada en este análisis. Otro hallazgo interesante es que la distribución espacial de las tasas de evacuación también coincide con Cutter et al. (2011). De este modo, el conglomerado sur registró una tasa de evacuación del $77,1 \%$, seguido por el conglomerado norte $(53,7 \%)$ y el conglomerado central (50,0 \%). Cutter et al. (2011) también observó una mayor predisposición

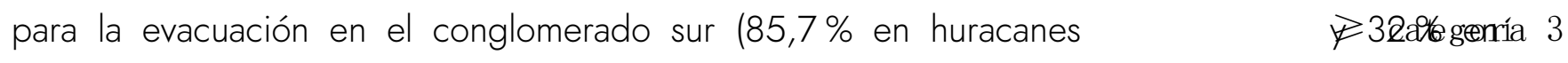
huracanes por debajo de este umbral) en comparación con el central $(74,2 \%-18,0 \%$ ) y el conglomerado norte $(70,7 \%-12,0 \%)$. Con cerca de 1,36 millones de personas viviendo en los 
condados costeros de Carolina del Sur (estimaciones de 2015), se calcula que 760000 personas evacuaron en respuesta al Huracán Matthew. Las estimaciones del número total de evacuados también se ajustan a otro de los métodos tradicionalmente empleados para monitorear y cuantificar evacuaciones, los sensores de tráfico. El Departamento de Tráfico de Carolina del Sur estimó que 355000 vehículos abandonaron los condados costeros durante el periodo comprendido entre el 4 y el 7 de octubre (SCDOT, 2017). El tamaño medio del hogar en Carolina del Sur varía entre 2,4 y 2,8 individuos, y contando con la experiencia previa de Cutter et al. (2011), que averiguaron el número de vehículos que las familias usarían en caso de evacuación, se estima que con una ocupación de 2.0 personas por vehículo el total de la población evacuada es de 710000 personas, lo que se sitúa próximo a nuestras estimaciones provenientes de Twitter.

Tabla 3. Tasas de evacuación y estimaciones de evacuados por conglomerado

\begin{tabular}{llllll}
\hline Conglomerado & Usuarios & Evacuados & \% Evacuados & Pob. 2015 & Evacuados estimados \\
\hline Sur & 105 & 81 & $77.10 \%$ & 245144 & 189111 \\
Central & 560 & 280 & $50.00 \%$ & 744526 & 372263 \\
Norte & 719 & 386 & $53.70 \%$ & 370497 & 198904 \\
\hline Total & 1384 & 747 & $54.00 \%$ & 1360167 & 760278 \\
\hline
\end{tabular}

Fuente: elaboración propia

\subsection{Estimación del porcentaje de desplazados / migrantes post-desastre}

Este estudio revela que el 36,4 \% de los usuarios de Twitter identificados abandonó Puerto Rico en las 15 semanas posteriores al huracán (hasta el 31 de diciembre) (Tabla 4). Los usuarios que viajaron fuera de Puerto Rico por un periodo igual o inferior a 4 semanas se consideraron no desplazados, ya que es probable que gran parte de estos viajes estuvieran relacionados con periodos vacacionales (Acción de Gracias y Navidad) o personas que buscaron refugio en los Estados Unidos (continentales) mientras los principales servicios básicos (electricidad, agua y teléfono) eran restablecidos en la isla. Siguiendo publicaciones previas (State et al., 2013), se consideró como desplazado únicamente a aquellos usuarios que estuvieron fuera de la isla un periodo superior a las 4 semanas, lo que corresponde a un $8.3 \%$ de la muestra.

La mayor parte de los desplazados abandonaron Puerto Rico en la primera mitad de Octubre (Figura 4), probablemente como consecuencia de la prolongada ausencia de servicio eléctrico. En total, un 76,3\% de los desplazados se marcharon de la isla en las primeras 6 semanas después de María. Este patrón coincide con los hallazgos al respecto de Hinojosa y Meléndez (2018). El 
proceso de retorno fue muy escalonado a lo largo de los meses posteriores, apreciándose mayores picos en los periodos postvacacionales y al final del año académico (mayo). De acuerdo con los datos, nueve meses después del desastre (hasta el 31 de mayo de 2018), solo el 54,6 \% de los desplazados habían retornado a Puerto Rico. Considerando la muestra completa, 3,8\% había abandonado la isla y no había regresado. Al cierre del estudio, la estimación más actualizada realizada por la Oficina del Censo de Estados Unidos reflejaba un desplazamiento de larga duración (migración) de aproximadamente 129848 personas, lo que se corresponde con un 3,9\% de la población total de Puerto Rico (US Census Bureau, 2018). Estos datos coinciden con las aproximaciones estimadas con datos de Twitter y sirven como relativa validación del método. Los resultados confirman por tanto que el Huracán María generó desplazados de larga duración. Sin embargo, es prematuro de momento vincular estos desplazados con migración permanente, lo que de ser realidad confirmaría los peores augurios para Puerto Rico (Stone, 2017).

Tabla 4. Duración de los viajes fuera de Puerto Rico después de María
de usuarios de Twitter residentes en Puerto Rico
\begin{tabular}{rrr} 
Usuarios & Porcentaje \\
\hline No abandonaron PR & 783 & $63.60 \%$ \\
Viajaron fuera de PR & 448 & $36.40 \%$ \\
4 semanas o menos & 326 & $26.50 \%$ \\
$5-12$ semanas & 24 & $1.90 \%$ \\
$13-24$ semanas & 24 & $1.90 \%$ \\
24 semanas o más & 55 & $4.50 \%$ \\
Duración desconocida & 19 & $1.50 \%$
\end{tabular}

Fuente: elaboración propia 


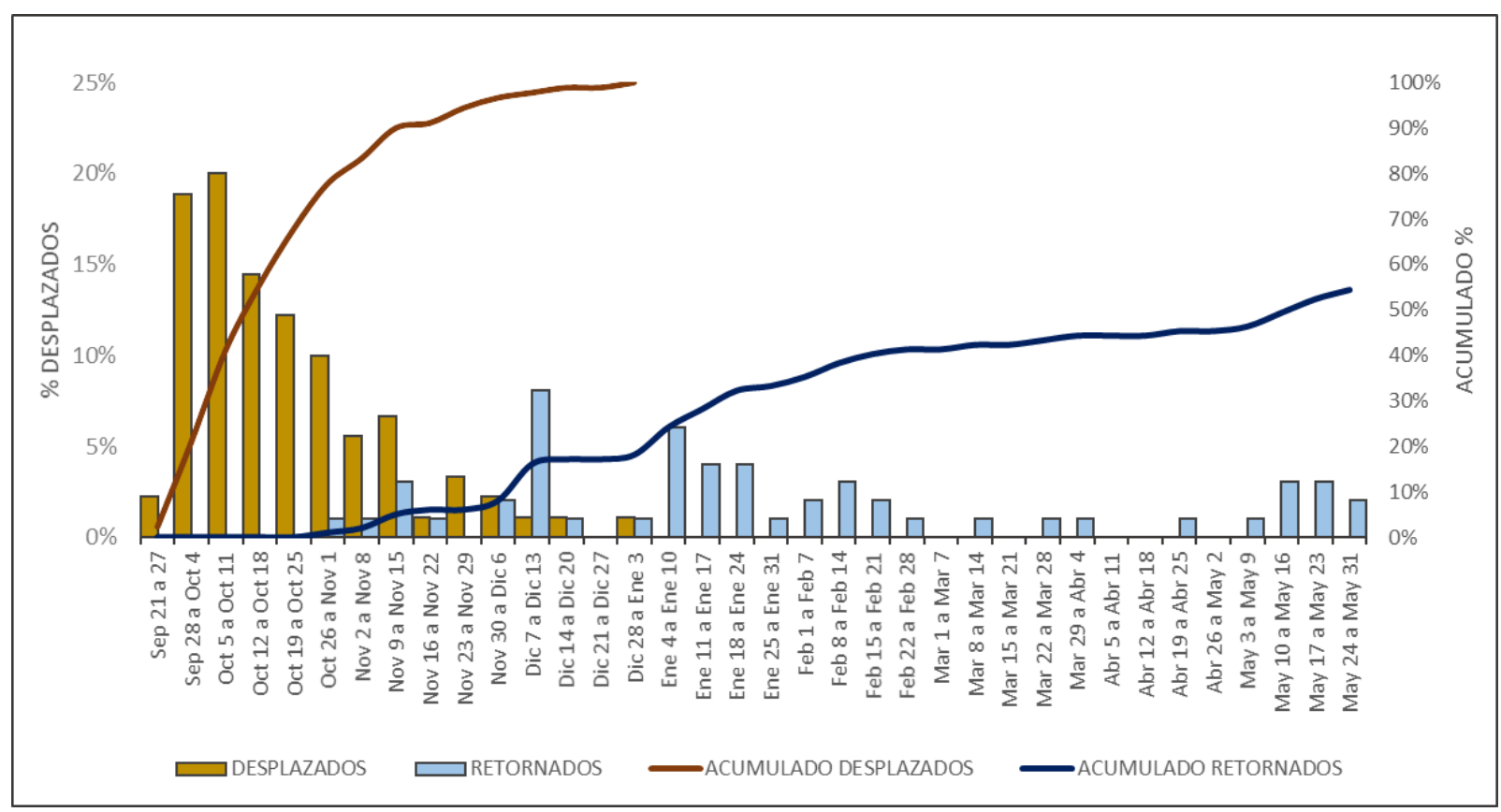

Fuente: elaboración propia

\subsection{Estimación de la afección de María en el número de visitantes}

La temporada de huracanes de 2017 dañó de forma muy significativa la ya de por sí precaria economía de Puerto Rico (Government of Puerto Rico, 2018). El turismo, una de las columnas en la que se sustenta la economía de la isla (Tourism Economics, 2019), recibió un profundo impacto como consecuencia de los largos periodos sin suministro eléctrico o de agua, el cierre de aeropuertos y puertos marítimos, y el daño por erosión y contaminación de las aguas en las playas. La Figura 5 compara el número de usuarios de Twitter no residentes identificados en cada semana del año anterior al Huracán María con los mismos datos para el año posterior a María. Esta gráfica constata que septiembre de 2017 comenzó con una cantidad de usuarios de Twitter no residentes superior a la registrada el año anterior, pero estos valores cayeron por debajo de los datos preMaría poco después del huracán (20 de septiembre de 2017). Sin embargo, la bajada fue menor de lo que se podría esperar y los valores se mantuvieron (en el conjunto de la isla) cercanos a los registrados en 2016-2017, muy probablemente como consecuencia del gran flujo de personal de emergencia desplazado desde el resto de Estados Unidos hasta la isla (Government of Puerto Rico, 2018). Los descensos respecto a los valores del año anterior fueron notablemente más acusados en los periodos de temporada alta, con descensos de alrededor del 30\% tanto en Acción de Gracias como en Navidad. El descenso durante las vacaciones de primavera (periodo vacacional de las universidades americanas y que atrae mucho turismo de playa y ocio nocturno a Puerto 
Rico) fue también alrededor del $30 \%$. En contra de lo esperado, una recuperación paulatina de la llegada de no residentes, los descensos más acusados se observan durante la segunda parte del verano, lo que coincide con la parte más activa de la temporada de huracanes en Puerto Rico, lo que ha sido relacionado con la preocupación de operadores turísticos y de visitantes particulares de que la isla no estuviera preparada para otra temporada activa (D'Ambrosio, 2018).

Figura 5. Media móvil de 3 semanas de usuarios de Twitter no residentes activos en Puerto Rico durante los periodos Sep. 2016 - Aug. 2017 (pre-desastre) y Sep. 2017 - Aug. 2018 (post-desastre)

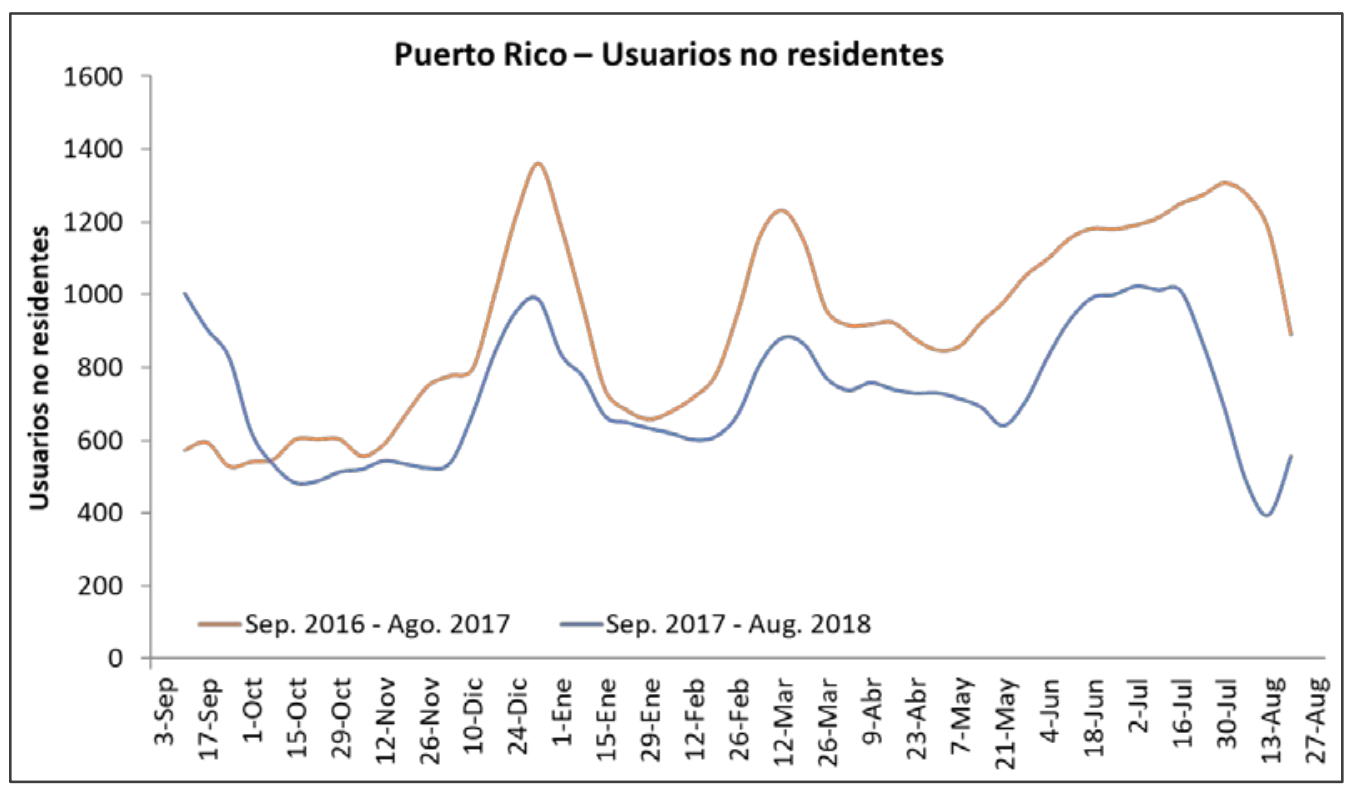

Fuente: elaboración propia

La variación en el número de usuarios visitantes no fue homogénea en toda la isla. Las zonas más afectadas por el huracán, el norte y este de la isla (Figura 6), lo que coincide con las zonas más turísticas de Puerto Rico, sufrieron los descensos más considerables. Las zonas central (Figure 5B), oeste (Figura 6D) y sur (Figura 6E) registraron descensos más comedidos o incluso balances anuales positivos. La zona norte recibió un $22 \%$ menos de visitantes (Figura 6C) entre octubre de 2017 y agosto de 2018, con los periodos de temporada no veraniegos con descensos de en torno al 30 \% y una marcada disminución de hasta el 60 \% al final del verano. La región este (Figura 6F), conocida por la calidad de sus playas, lo que atrae a un gran número de turistas durante todo el año, experimentó un patrón muy similar a la zona norte de la isla y perdió a lo largo del año postMaría un tercio de sus visitantes. La región oeste cerró el año posterior a María con un descenso del 15\% en la llegada usuarios visitantes, pero logró dos periodos de incremento respecto al año anterior. El primero de ellos, justo después del huracán, se explica por la llegada de operarios de 
emergencia, mientras que el segundo, durante las vacaciones de primavera, se puede relacionar con un traslado de turismo desde la zona más afectada y turística (este) hacia las costas occidentales. La región sur fue la única que experimentó un incremento anual en el número de usuarios de Twitter no residentes (11\%), ya que esta zona se erigió como el centro de operaciones de recuperación de la isla. Es por ello por lo que los incrementos de visitantes se centraron en periodos no vacacionales y que este porcentaje de usuarios no residentes decreció con el paso de los meses, aunque conclusiones más fiables deberían extraerse en un análisis de una serie temporal más larga.

Figura 6. Porcentaje de variación de usuarios de Twitter no-residentes en Puerto Rico durante el periodo del 1 de septiembre de 2017 al 31 de agosto de 2018 respecto al año pre-desastre (1 de septiembre de 2016 a 31 de agosto de 2017)
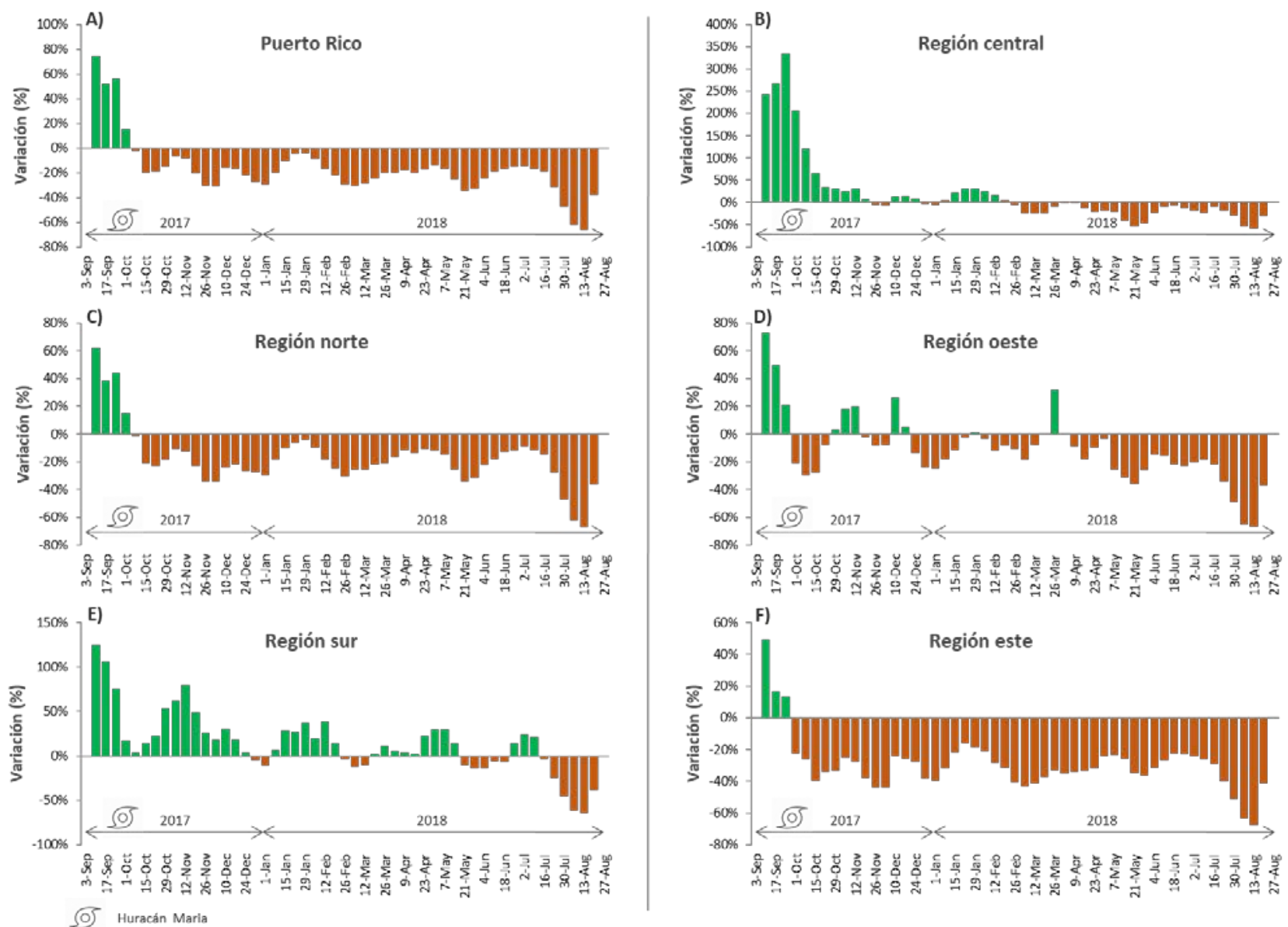

Nota: En verde se muestran las variaciones positivas y en rojo las variaciones negativas.

Fuente: elaboración propia 


\subsection{Twitter como muestra de la sociedad}

Una de las principales preocupaciones al utilizar datos provenientes de Twitter es que podría no reflejar de forma acertada el conjunto de la sociedad en lo que respecta la composición racial o étnica, al sexo, al estatus socioeconómico o a la edad. Existe una profusa y emergente bibliografía al respecto que discute el perfil demográfico de los usuarios de Twitter (Mislove et al., 2011; Hecht \& Stephens, 2014). Estos estudios han encontrado un sesgo urbano, masculino, y de población blanca, lo que podría infrarrepresentar algunos grupos de la población. El problema de la representatividad de las muestras es algo que se comparte con las encuestas, ya que estas tienden a sobrerrepresentar a grupos de personas por encima de los 60 años, personas con mayor grado educativo, y de grupos raciales mayoritarios.

Esto mismo se observó en Martín et al. (2019), donde se realizó una encuesta para conocer el comportamiento de la población durante la evacuación del Huracán Matthew. De las 19829 encuestas enviadas por correo ordinario (dirigiendo a los ciudadanos a una plataforma electrónica donde responder a las preguntas), únicamente 329 hogares respondieron (menos de un $2 \%$ ). Al ya escaso porcentaje de respuesta hay que añadir que la muestra presentó un gran sesgo en cuanto a la raza (mayoritariamente raza blanca) y a la edad (personas mayores). La Figura 7 muestra como la mayor parte de las respuestas a la encuesta vino de parte de mayores de 65 años. Este es un gran ejemplo de la necesidad de encontrar métodos alternativos para conocer el comportamiento espacial de la población y de cómo la sombra digital puede ser una de las soluciones.

En lo que respecta a Twitter, la Figura 7 muestra una sobrerrepresentación de los individuos jóvenes. Se aprecia una asimetría en el sexo de los usuarios de Twitter, con un 60,7\% de los usuarios siendo mujeres (muy marcada entre los más jóvenes), lo que se distancia de la sobrerrepresentación masculina encontrada en otros estudios (Mislove et al., 2011) pero que confirma más recientes análisis (liang et al., 2018). Respecto a la raza, Twitter $(72,1 \%$ de población blanca) se asemeja más a la distribución racial de la población (66,7\% población blanca) que la encuesta (86,3 \% población blanca), aunque todavía existe un ligero sesgo hacia la mayoría racial. Analizando conjuntamente las muestras de Twitter y las de la encuesta podemos observar cómo se complementan muy positivamente, ya que se recibe información de grupos distintos, encontrando únicamente infrarrepresentación en el grupo entre 45 y 64 años (en esencia y en términos generales, demasiado mayores para Twitter y demasiado jóvenes para responder a encuestas). 
Figura 7. Comparación de la distribución de edad y sexo entre las muestras de la encuesta y de Twitter y relación con la distribución de la población de los condados costeros de Carolina del Sur

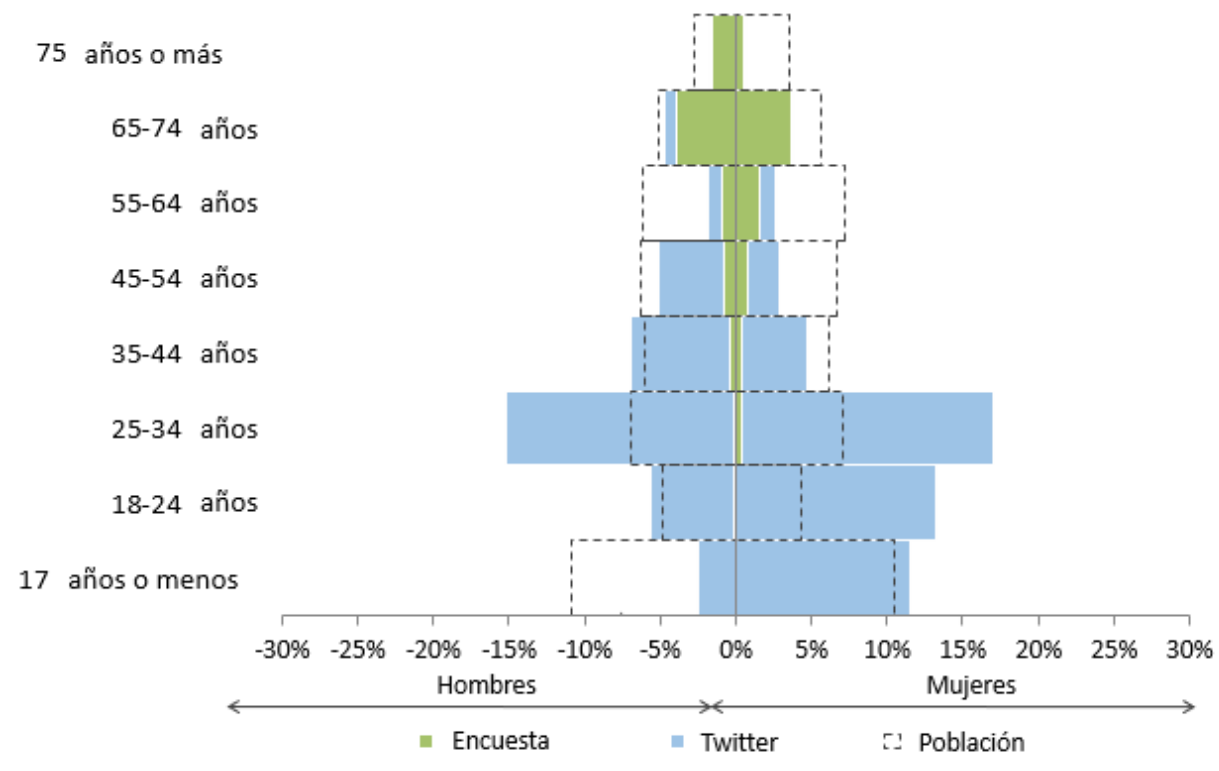

Fuente: Encuesta Americana de Comunidades (2016)

\section{Conclusiones}

El estudio de los movimientos poblacionales ha sido siempre un gran reto para los investigadores, particularmente limitados por la ausencia de datos públicos tanto de movimientos de corta duración como de movimientos de larga duración o permanentes (Spyratos et al., 2018). El desarrollo del transporte moderno y la consiguiente aceleración de la movilidad añade complejidad al estudio de los movimientos de la población humana. El estudio de movimientos poblacionales desencadenados por emergencias y desastres es una tarea todavía más compleja (Laczko, 2015; Rango \& Vespe, 2017). Uno de los grandes problemas se relaciona con la rápida secuencia de movimientos durante desastres, lo que generalmente escapa las medidas temporalmente dispersas de las estadísticas de población oficiales (censos), con una periodicidad bianual en el mejor de los casos. De este modo, dar respuesta a respuestas básicas ("¿cuántos?", "¿dónde?", “¿cuándo?", "¿quién?", o “¿por qué?") se hace muy complicado.

La llegada de las tecnologías digitales que tanto ha cambiado la sociedad en las últimas décadas se ha visto como una oportunidad desde el campo del comportamiento espacial, provocando que muchos investigadores estén activamente explorando la utilización de la sombra digital geoespacial en sus investigaciones (Isaacson \& Shoval, 2006). Muchos coinciden en el potencial de estos datos generados por la actividad digital diaria de las personas, pero muchas cuestiones referentes 
a su validez, representatividad, y aplicaciones permanecen todavía inexploradas o sin respuesta. Aunque la aplicación de datos de sensores ciudadanos pasivos está creciendo exponencialmente en muchas disciplinas, su aplicación en el campo de los riesgos es todavía débil y sólo desde la epidemiología se han explotado estas nuevas posibilidades de forma sistemática.

La investigación presentada en este artículo responde por tanto a los llamamientos por la innovación en el campo del comportamiento espacial, particularmente en situaciones de emergencia, y a la ausencia de estudios en profundidad acerca de la validez de la sombra digital geoespacial para el monitoreo de los movimientos poblacionales inducidos por riesgos naturales. Los resultados de este conjunto de estudios individuales confirman el potencial de tweets georreferenciados como complemento de métodos tradicionales para el estudio del comportamiento espacial, destacando al mismo tiempo las limitaciones de estos nuevos enfoques.

La gran debilidad de la sombra digital geoespacial de la red Twitter surge cuando se intenta analizar preguntas acerca de la motivación del desplazamiento ("¿por qué?) o de las características del desplazado/evacuado ("¿quién?"). Aunque las investigaciones han demostrado que estos datos pueden ser de utilidad para incluir grupos que las encuestas tienen dificultad en captar (jóvenes, minorías raciales, o poblaciones flotantes), la variedad y confianza acerca de las características demográficas que se puede extraer de los perfiles de Twitter es baja en comparación a las encuestas (dónde el encuestado responde a preguntas directas de estas cuestiones). Por otra parte, la asociación de los movimientos poblacionales con riesgos naturales estudiados se basa en inferencias y no se tiene hasta el momento pruebas empíricas que validen estas suposiciones.

En general, la sombra espacial de la red social Twitter si se ha mostrado adecuada para el seguimiento de un porcentaje relevante de individuos durante las fases de respuesta y recuperación de un huracán. El enfoque basado en Twitter permite estimaciones relativamente precisas de la magnitud ("¿cuántos?"), momento ("¿cuándo?"), y destino ("¿dónde?") en evacuaciones (Martín et al., 2017; Martín et al., 2019) y desplazamientos post-desastre a una escala igual o superior al condado, así como de la llegada de visitantes a zonas afectadas. Aunque se trate de datos con una periodicidad y una resolución espacial limitada, se han mostrado suficientes para medir movimientos poblaciones de esta escala espacio-temporal. Siguiendo está lógica, el método podría tener validez para el monitoreo de movimientos de poblaciones derivados de otros fenómenos de gran escala como terremotos, tsunamis, volcanes, sequías o grandes inundaciones. Por el contrario, el estudio de fenómenos de pequeña escala (e.g., 
tornados o ataques terroristas), en los que las evacuaciones o desplazamientos no son de gran distancia, no puede ser estudiado a través de la geolocalización de los tweets y requiere de otras fuentes de datos ciudadanos pasivos como los registros de teléfonos móviles.

Agradecimientos: El autor quiere agradecer a la Comisión Fulbright y a la Fundación Iberdrola por la financiación aportada para la consecución del doctorado del que deriva esta publicación.

Declaración responsable: El autor declara que no hay conflicto de intereses existente en relación a la publicación de este artículo. 


\section{Bibliografía}

Adey, P. (2016). Emergency mobilities. Mobilities, 11(1), 3248. https://doi.org/10.1080/17450101.2015.1103533

Adger, W. N., de Campos, R. S., \& Mortreux, C. (2018). Mobility, displacement and migration, and their interactions with vulnerability and adaptation to environmental risks. In R. McLeman \& F. Gemenne (Eds.), Routledge Handbook of Environmental Displacement and Migration (pp. 29-41). New York: Routledge. https://doi.org/10.4324/9781315638843-3

Amini, A., Kung, K., Kang, C., Sobolevsky, S., \& Ratti, C. (2014). The impact of social segregation on human mobility in developing and industrialized regions. EPJ Data Science, 3(1), 120. https://doi.org/10.1140/epjds31

Andrienko, N., Andrienko, G., Stange, H., Liebig, T., \& Hecker, D. (2012). Visual analytics for understanding spatial situations from episodic movement data. KI-Künstliche Intelligenz, 26(3), 241251. https://doi.org/10.1007/s13218-012-0177-4

Archibald, E., \& McNeil, S. (2012). Learning from traffic data collected before, during and after a hurricane. IATSS Research, 36(1), 1-10. https://doi.org/10.1016/j.iatssr.2012.06.002

Arlikatti, S., Lindell, M. K., Prater, C. S., \& Zhang, Y. (2006). Risk area accuracy and hurricane evacuation expectations of coastal residents. Environment and Behavior, 38(2), 226247. https://doi.org/10.1177/0013916505277603

Baker, E. J. (2009). Acquiring data on travel behaviour during emergencies and exceptional events. In P. Bonnel, M. Lee-Gosselin, J. Zmud, \& J. L. Madre (Eds.), Transport Survey Methods: Keeping up with a Changing World (pp. 197-211). Bingley: Emerald Group Publishing Limited. https://doi.org/10.1108/9781848558458-011

Bajardi, P., Delfino, M., Panisson, A., Petri, G., \& Tizzoni, M. (2015). Unveiling patterns of international communities in a global city using mobile phone data. EPJ Data Science, 4(3), 117. https://doi.org/10.1140/epjds/s13688-015-0041-5

Bauman, Z. (1996). From pilgrim to tourist-or a short history of identity. Questions of Cultural Identity, 1, 18-36. https://doi.org/10.4135/9781446221907.n2

Bengtsson, L., Lu, X., Thorson, A., Garfield, R., \& Von Schreeb, J. (2011). Improved response to disasters and outbreaks by tracking population movements with mobile phone network data: A post- 
earthquake geospatial study in Haiti. PLoS Medicine, 8(8), e1001083. https://doi.org/10.1371/journal.pmed.1001083

Birenboim, A., \& Shoval, N. (2016). Mobility research in the age of the smartphone. Annals of the American Association of Geographers, 106(2), 283291. https://doi.org/10.1080/00045608.2015.1100058

Black, R., Arnell, N. W., Adger, W. N., Thomas, D., \& Geddes, A. (2013). Migration, immobility and displacement outcomes following extreme events. Environmental Science \& Policy, 27, S32S43. https://doi.org/10.1016/j.envsci.2012.09.001

Bowser, G. C. (2013). Determining the differences in hurricane perception and evacuation behavior in the elderly of South Carolina (Doctoral dissertation). University of South Carolina, Columbia, SC, USA. Retrieved from https://scholarcommons.sc.edu/etd/2534

Brommer, D. M., \& Senkbeil, J. C. (2010). Pre-landfall evacuee perception of the meteorological hazards associated with Hurricane Gustav. Natural Hazards, 55(2), 353369. https://doi.org/10.1007/s11069-010-9532-7

Bowser, G. C., \& Cutter, S. L. (2015). Stay or go? Examining decision making and behavior in hurricane evacuations. Environment: Science and Policy for Sustainable Development, 57(6), 2841. https://doi.org/10.1080/00139157.2015.1089145

Burton, I., \& Hewitt, K. (1971). The hazardousness of a place: a regional ecology of damaging events. Toronto: University of Toronto Press.

Burton, S. H., Tanner, K. W., Giraud-Carrier, C. G., West, J. H., \& Barnes, M. D. (2012). "Right time, right place" health communication on Twitter: Value and accuracy of location information. Journal of Medical Internet Research, 14(6). https://doi.org/10.2196/jmir.2121

Chaintreau, A., Hui, P., Crowcroft, J., Diot, C., Gass, R., \& Scott, J. (2007). Impact of human mobility on opportunistic forwarding algorithms. IEEE Transactions on Mobile Computing, 6, 606620. https://doi.org/10.1109/infocom.2006.172

Çolak, S., Lima, A., \& González, M. C. (2016). Understanding congested travel in urban areas. Nature Communications, 7, 10793. https://doi.org/10.1038/ncomms10793

Cooper, M., Biehl, J., Filby, G., \& Kratz, S. (2016). LoCo: Boosting for indoor location classification combining Wi-Fi and BLE. Personal and Ubiquitous Computing, 20(1), 8396. https://doi.org/10.1007/s00779-015-0899-z 
Cresswell, T. (2011). Mobilities I: Catching up. Progress in Human Geography, 35(4), 550558. https://doi.org/10.1177/0309132510383348

Cutter, S. L., Emrich, C. T., Bowser, G. C., Angelo, D., \& Mitchell, J. T. (2011). South Carolina hurricane behacuation study. Retrieved from hittp://webra.cas.sc.edu/hvri/docs/HES_2011_Final_Report.pdf

D’Ambrosio, R. (2018, May 14). As hurricane season nears, travel agents wonder if Puerto Rico is ready. Travelmarket. Retrieved from https://www.travelmarketreport.com/articles/As-HurricaneSeason-Nears-Travel-Agents-Wonder-if-Puerto-Rico-Is-Ready

Dow, K., \& Cutter, S. L. (1998). Crying wolf: Repeat responses to hurricane evacuation orders. Coastal Management, 26(4), 237-52. https://doi.org/10.1080/08920759809362356

Dow, K., \& Cutter, S. L. (2000). Public orders and personal opinions: Household strategies for hurricane risk assessment. Global Environmental Change Part B: Environmental Hazards, 2(4), 143-155. https://doi.org/10.1016/S1464-2867(01)00014-6

Dow, K., \& Cutter, S. L. (2002). Emerging hurricane evacuation issues: Hurricane Floyd and South Carolina. Natural Hazards Review, 3(1), 12-18. https://doi.org/10.1061/(ASCE)15276988(2002)3:1(12)

Dueker, D., Taher, M., Wilson, J., \& McConnell, R. (2014). Evaluating children's location using a personal GPS logging instrument: limitations and lessons learned. Journal of Exposure Science and Environmental Epidemiology, 24(3), 244. https://doi.org/10.1038/jes.2013.11

Durage, S. W., Kattan, L., Wirasinghe, S. C., \& Ruwanpura, J. Y. (2014). Evacuation behaviour of households and drivers during a tornado. Natural Hazards, 71(3), 14951517. https://doi.org/10.1007/s11069-013-0958-6.

Echenique, M., \& Melgar, L. (2018, May 11). Mapping Puerto Rico's hurricane migration with mobile phone CityLab. Retrieved from https://www.citylab.com/environment/2018/05/watch-puerto-ricos-hurricane-migration-viamobile-phone-data/559889/?utm_source=SFFB

Elgethun, K., Yost, M. G., Fitzpatrick, C. T., Nyerges, T. L., \& Fenske, R. A. (2007). Comparison of Global Positioning System (GPS) Tracking and Parent-Report Diaries to Characterize Children's Time-Location Patterns. Journal of Exposure Science \& Environmental Epidemiology, 17, 196206. https://doi.org/10.1038/sj.jes.7500496

Estébanez Álvarez, J. (1990) Tendencias y problemática actual de la geografía. Madrid: Cincel. 
Falcón-Lezama, J. A., Santos-Luna, R., Román-Pérez, S., Martínez-Vega, R. A., Herrera-Valdez, M. A., Kuri-Morales, Á. F., ... \& Ramos-Castañeda, J. (2017). Analysis of spatial mobility in subjects from a Dengue endemic urban locality in Morelos State, Mexico. PloS ONE, 12(2), e0172313. https://doi.org/10.1371/journal.pone.0172313

Finch, C., Emrich, C. T., \& Cutter, S. L. (2010). Disaster disparities and differential recovery in New Orleans. Population and Environment, 31(4), 179-202. https://doi.org/10.1007/s11111$\underline{009-0099-8}$

Fussell, E., Sastry, N., \& VanLandingham, M. (2010). Race, socioeconomic status, and return migration to New Orleans after Hurricane Katrina. Population and Environment, 37(1-3), 2042. https://doi.org/10.1007/s11111-009-0092-2

Gerber, M. S. (2014). Predicting crime using Twitter and kernel density estimation. Decision Support Systems, 61, 115-125. https://doi.org/10.1016/j.dss.2014.02.003

Gething, P. W., \& Tatem, A. J. (2011). Can mobile phone data improve emergency response to natural disasters?. PLoS medicine, 8(8),

e1001085. https://doi.org/10.1371/journal.pmed.1001085

Gladwin, H., \& Peacock W. G. (1997) Warning and evacuation: A night for hard houses. In W. G. Peacock, B. Morrow \& H. Gladwin (Eds.) Hurricane Andrew: Gender, ethnicity and the sociology of disasters (pp. 52-74). New York: Routledge.

Golledge, R. G., \& Stimson, R. J. (1997). Spatial behavior: A geographic perspective. London: The Guilford Press.

Gong, Y., Lin, Y., \& Duan, Z. (2017). Exploring the spatiotemporal structure of dynamic urban space using metro smart card records. Computers, Environment and Urban Systems, 64, 169183. https://doi.org/10.1016/j.compenvurbsys.2017.02.003

González, M. C., Hidalgo, C. A., \& Barabási, A.-L., (2008). Understanding individual human mobility patterns. Nature, 453, 779-782. https://doi.org/10.1038/nature06958

Goodchild, M. F. (2007). Citizens as sensors: The world of volunteered geography. Geofournal, 69(4), 211-221. https://doi.org/10.1007/s10708-007-9111-y

Government of Puerto Rico. (2018). Puerto Rico disaster recovery action plan. Retrieved from http://www.cdbg-dr.pr.gov/wp-content/uploads/2018/07/HUD-Approved-ActionPlan_EN.pdf 
Groen, J. A., \& Polivka, A. E. (2010). Going home after Hurricane Katrina: Determinants of return migration and changes in affected areas. Demography, 47(4), 821844. https://doi.org/10.1007/BF03214587

Harvey, D. (1989). The Condition of Postmodernity. An Enquiry into the Origins of Cultural Change. Oxford: Blackwell.

Hecht, B. J., \& Stephens, M. (2014). A tale of cities: Urban biases in volunteered geographic information. ICWSM, 14, 197-205.

Hinojosa, J., \& Meléndez, E. (2018). Puerto Rican exodus: One year since Hurricane Maria. Retrieved from https://centropr.hunter.cuny.edu/sites/default/files/RB201805_SEPT2018\%20\%281\%29.pdf

Isaacson, M., \& Shoval, N. (2006). Application of tracking technologies to the study of pedestrian spatial behavior. The Professional Geographer, 58(2), 172-183. https://doi.org/10.1111/j.1467$\underline{9272.2006 .00524 . x}$

Jiang, Y., Li, Z., \& Ye, X. (2018). Understanding demographic and socioeconomic biases of geotagged Twitter users at the county level. Cartography and Geographic Information Science, 115. https://doi.org/10.1080/15230406.2018.1434834

Johnson, T. P., \& Wislar, J. S. (2012). Response rates and nonresponse errors in surveys. Journal of the American Medical Association, 307(17), 18051806. https://doi.org/10.1001/jama.2012.3532

Jurdak, R., Zhao, K., Liu, J., Aboulaoude, M., Cameron, M., \& Newth, D. (2015). Understanding human mobility from Twitter. PLoS ONE, e0131469. https://doi.org/10.1371/journal.pone.0131469

Kates, R. W. 1962. Hazard and choice perception in flood plain management (University of Chicago, Department of Geography Research Paper, No. 78).

Kishore, N., Marqués, D., Mahmud, A., Kiang, M. V., Rodriguez, I., Fuller, A., ... Buckee, C.O. (2018). Mortality in Puerto Rico after Hurricane Maria. New England Journal of Medicine, 379, 162-170. https://doi.org/10.1056/NEJMsa1803972

Kontokosta, C. E., \& Johnson, N. (2017). Urban phenology: Toward a real-time census of the city using Wi-Fi data. Computers, Environment and Urban Systems, 64, 144153. https://doi.org/10.1016/j.compenvurbsys.2017.01.011 
Kumar, D., \& Ukkusuri, S. V. (2018, April). Utilizing geo-tagged tweets to understand evacuation dynamics during emergencies: A case study of Hurricane Sandy. In Companion Proceedings of the Web Conference 2018 (pp. 1613-1620). Lyon, France. hittps://doi.org/10.1145/3184558.3191619

Laczko, F. (2015). Factoring migration into the development data revolution. Journal of International Affairs, 68(2), 1.

Li, Z., Wang, C., Emrich, C. T., \& Guo, D. (2018). A novel approach to leveraging social media for rapid flood mapping: A case study of the 2015 South Carolina floods. Cartography and Geographic Information Science, 45(2), 97110. https://doi.org/10.1080/15230406.2016.1271356

Lima, V. V., Miranda, J. M., Baptista, M. A., Catalão, J., González Rodríguez, E. M., Otero, L., ... \& Carreño Herrero, E. (2010). Impact of a 1755-like tsunami in Huelva, Spain. Natural Hazards and Earth System Science, 10, 139-148. https://doi.org/10.5194/nhess-10-139-2010

Lindell, M. K., Kang, J. E., \& Prater, C. S. (2011). The logistics of household hurricane evacuation. Natural Hazards, 58(3), 1093-1109. https://doi.org/10.1007/s11069-011-9715-x

Manning, P. (2012). Migration in world history. New York: Routledge.

Martín, Y., Li, Z., \& Cutter, S. L. (2017). Leveraging Twitter to gauge evacuation compliance: Spatiotemporal analysis of Hurricane Matthew. PLoS ONE, 12, e0181701. https://doi.org/10.1371/journal.pone.0181701

Martín, Y., Cutter, S. L., \& Li, Z. (2020). Bridging social media and survey data for the evacuation assessment of Hurricane Matthew and Hurricane Irma. Natural Hazards Review, 21(2). https://doi.org/10.1061/(ASCE)NH.1527-6996.0000354

McLeman, R., \& Gemenne, F. (Eds.). (2018). Routledge handbook of environmental displacement and migration. Routledge. https://doi.org/10.4324/9781315638843

Mislove, A., Lehmann, S., Ahn, Y. Y., Onnela, J. P., \& Rosenquist, J. N. (2011). Understanding the demographics of Twitter users. In Proceedings of the Fifth International AAAI Conference on Weblogs and Social Media. Barcelona, Spain. Retrieved from http://www.aaai.org/ocs/index.php/ICWSM/ICWSM11/paper/viewFile/2816/3234

Mitchell, J. T., Cutter, S. L., \& Edmonds, A. S. (2007). Improving shadow evacuation management: Case study of the Graniteville, South Carolina chlorine spill. Journal of Emergency Management, 5(1), 28-34. https://doi.org/10.5055/jem.2007.0041 
Moon, H., Kim, C., \& Lee, W. (2016). A UAV based 3-D positioning framework for detecting locations of buried persons in collapsed disaster area. international archives of the photogrammetry, Remote Sensing and Spatial Information Sciences, 41. https://doi.org/10.5194/isprsarchives-XLI-B8-121-2016

National Hurricane Center (NHC). (2018). NHC Data in GIS Formats. Retrieved from https://www.nhc.noaa.gov/gis/

National Institute of Standards and Technology (NIST). (2017). Hurricane Maria (2017): Preliminary Peak Wind Gust. Retrieved from https://www.nist.gov/sites/default/files/documents/2018/02/20/03_update_on_prelimi nary_r econnaissance_of_hurricane_maria_puerto_rico.pdf

Pasch, R., Penny, A., \& Berg, R. (2018). Hurricane Maria (AL 152017). Retrieved from https://www.nhc.noaa.gov/data/tcr/AL152017_Maria.pdf

Paz-Soldan, V. A., Reiner Jr, R. C., Morrison, A. C., Stoddard, S. T., Kitron, U., Scott, T. W., ... \& Vazquez-Prokopec, G. M. (2014). Strengths and weaknesses of Global Positioning System (GPS) data-loggers and semi-structured interviews for capturing fine-scale human mobility: findings from lquitos, Peru. PLoS neglected tropical 8(6), e2888. https://doi.org/10.1371/journal.pntd.0002888

Perttunen, M., Kostakos, V., Riekki, J., \& Ojala, T. (2014). Spatio-temporal patterns link your digital identities. Computers, Environment and Urban Systems, 47, 5867. https://doi.org/10.1016/j.compenvurbsys.2013.12.004

Phithakkitnukoon, S., Smoreda, Z., \& Olivier, P. (2012). Socio-geography of human mobility: A study using longitudinal mobile phone data. PloS one, 7(6), e39253. https://doi.org/10.1371/journal.pone.0039253

Rango, M., \& Vespe, M. (2017). Big Data and Alternative Data Sources on Migration: From CaseStudies to Policy Support (Summary report European Commission - Joint Research Centre) Retrieved from https://gmdac.iom.int/big-data-and-alternative-data-sources-on-migration-from-casestudies-to-policy-support

San Carlos Arce, R., Onuki, M., Esteban, M., \& Shibayama, T. (2017). Risk awareness and intended tsunami evacuation behaviour of international tourists in Kamakura City, Japan. International Journal of Disaster Risk Reduction, 23, 178-92. https://doi.org/10.1016/j.ijdrr.2017.04.005 
Shareck, M., Kestens, Y., \& Gauvin, L. (2013). Examining the spatial congruence between data obtained with a novel activity location questionnaire, continuous GPS tracking, \& prompted recall surveys. International Journal of Health Geographics, 12(40). https://doi.org/10.1186/1476-072X$\underline{12-40}$

Shoval, N., McKercher, B., Ng, E., \& Birenboim, A. (2011). Hotel location and tourist activity in cities. Annals of Tourism Research, 38(4), 15941612. https://doi.org/10.1016/j.annals.2011.02.007

Siebeneck, L. K., \& Cova, T. J. (2012). Spatial and temporal variation in evacuee risk perception throughout the evacuation and return-entry process. Risk Analysis, 32(9), 146880. https://doi.org/10.1111/15.1539-6924.2011.01781.x

Slovic, P. (1987). Perception of risk. Science, 236(4799), 28085. https://doi.org/10.1126/science.3563507

Sorensen, J. H., \& Mileti, D. S. (1988). Warning and evacuation: Answering some basic questions. Industrial Crisis Quarterly, 2, 195-209. https://doi.org/10.1177/108602668800200302

Smith, S. K., \& McCarty, C. (2009). Fleeing the storm(s): An examination of evacuation behavior during Florida's 2004 hurricane season. Demography, 46, 127145. https://doi.org/10.1353/dem.0.0048

Sobolevsky, S., Sitko, I., Des Combes, R. T., Hawelka, B., Arias, J. M., \& Ratti, C. (2014). Money on the move: Big data of bank card transactions as the new proxy for human mobility patterns and regional delineation. the case of residents and foreign visitors in Spain. In Proceedings of the 2014 IEEE International Congress on Big Data (pp. 136-143). IEEE. Washington, DC, USA. https://doi.org/10.1109/BigData.Congress.2014.28

Solares, J. M., \& Arroyo, A. L. (2004). The great historical 1755 earthquake. Effects and damage in Spain. Journal of Seismology, 8(2), 275-294.

South Carolina Department of Transportation (SCDOT). (2017). Average annual daily traffic. Retrieved from https://www.scdot.org/default.aspx

Spence, P. R., Lachlan, K. A., \& Rainear, A. M. (2016). Social media and crisis research: Data collection and directions. Computers in Human Behavior, 54, 667672. https://doi.org/10.1016/j.chb.2015.08.045 
Spyratos, S., Vespe, M., Natale, F., Ingmar, W., Zagheni, E., \& and Rango, M. (2018). Migration data using social media: A European perspective (EUR 29273 EN). https://doi.org/10.2760/964282

Stanton, M. C., Yamauchi, M., Mkwanda, S. Z., Ndhlovu, P., Matipula, D. E., Mackenzie, C., \& Kelly-Hope, L. A. (2017). Measuring the physical and economic impact of filarial lymphoedema in Chikwawa district, Malawi: A case-control study. Infectious Diseases of Poverty, 6(1), 28. https://doi.org/10.1186/s40249-017-0241-2

State, B., Weber, I., \& Zagheni, E. (2013, February). Studying inter-national mobility through IP geolocation. In Proceedings of the Sixth ACM International Conference on Web Search and Data Mining. (pp. 265-274). ACM. Rome, Italy. https://doi.org/10.1145/2433396.2433432

Stewart, S. R. (2017). Hurricane Matthew (AL 142016). Retrieved from http://www.nhc.noaa.gov/data/tcr/AL142016_Matthew.pdf

Stone, L. (2017). How low will Puerto Rico's population go? Retrieved from https://medium.com/migration-issues/how-low-will-puerto-ricos-population-go-

c8d108ac8b3b

Tamima, U., \& Chouinard, L. (2016). Development of evacuation models for moderate seismic zones: A case study of Montreal. International Journal of Disaster Risk Reduction, 16, 16779. https://doi.org/10.1016/j.ijdrr.2016.02.003

Tourism Economics. (2019). Economic Impacts of Visitor Spending in Puerto Rico. Retrieved from hittp://www.puertoricodmo.com/DMOLanding/wp-content/uploads/2019/05/AnalysisPuerto-Rico-Visitor-Economic-Impact.pdf

Ukkusuri, S. V., Zhan, X., Sadri, A. M., \& Ye, Q. (2014). Use of social media data to explore crisis informatics: Study of 2013 Oklahoma Tornado. Transportation Research Record, 2459(1), 110_ 118. https://doi.org/10.3141/2459-13

United States Census Bureau (2018). December 19, 2018. Release number CB18-193. Retrieved from https://www.census.gov/newsroom/press-releases/2018/estimates-national-state.html

Vazquez-Prokopec, G. M., Stoddard, S. T., Paz-Soldan, V., Morrison, A. C., Elder, J. P., Kochel, T. J., Scott, T.W., \& Kitron, U. (2009). Usefulness of commercially available GPS data-loggers for tracking human movement and exposure to dengue virus. International Journal of Health Geographics, 8(1), 68. https://doi.org/10.1186/1476-072x-8-68 
Vogel, N., Theisen, C., Leidig, J. P., Scripps, J., Graham, D. H., \& Wolffe, G. (2015). mining mobile datasets to enable the fine-grained stochastic simulation of ebola diffusion. Procedia Computer Science, 51, 765-774. https://doi.org/10.1016/j.procs.2015.05.197

Wang, H., Zhang, X., Wu, L., Hou, C., Gong, H., Zhang, Q., \& Ouyang, M. (2015). Beijing passenger car travel survey: Implications for alternative fuel vehicle deployment. Mitigation and Adaptation Strategies for Global Change, 20(5), 817-835. https://doi.org/10.1007/s11027-0149609-9

Wang, Q., \& Taylor, J. E. (2014). Quantifying, comparing human mobility perturbation during Hurricane Sandy, Typhoon Wipha, Typhoon Haiyan. Procedia Economics and Finance, 18, 3338. https://doi.org/10.1016/S2212-5671(14)00910-1

Wang, Q., \& Taylor, J. E. (2016). Patterns and limitations of urban human mobility resilience under the influence of multiple types of natural disaster. PLOS ONE, 17(1), e0147299. hittps://doi.org/10.1371/journal.pone.0147299

Wesolowski, A., Eagle, N., Tatem, A. J., Smith, D. L., Noor, A. M., Snow, R. W., \& Buckee, C. O. (2012). Quantifying the impact of human mobility on malaria. Science, 338(6104), 267270. https://doi.org/10.1126/science. 1223467

White, G. F. (1945). Human adjustments to floods: A geographical approach to the flood problem in the United States. Research Paper No. 29. Chicago: Department of Geography, University of Chicago. Retrieved from

https://biotech.law.Isu.edu/Climate/docs/Human_Adj_Floods_White.pdf

Widener, M. J., \& Li, W. (2014). Using geolocated Twitter data to monitor the prevalence of healthy and unhealthy food references across the US. Applied Geography, 54, 189197. https://doi.org/10.1016/j.apgeog.2014.07.017

Widhalm, P., Yang, Y., Ulm, M., Athavale, S., \& González, M. C. (2015). Discovering urban activity patterns in cell phone data. Transportation, 42(4), 597623. https://doi.org/10.1007/s11116-015-9598-x

Willoughby, H. E. (2012). Distributions and trends of death and destruction from hurricanes in the United States, 1900-2008. Natural Hazards Review, 13(1), 57-64. doi:10.1061/(ASCE)NH.1527-6996.0000046

Wolshon, B., \& Pande, A. (2016). Traffic Engineering Handbook. John Wiley \& Sons. 
Yabe, T., Sekimoto, Y., Tsubouchi, K., \& Ikemoto, S. (2019). Cross-comparative analysis of evacuation behavior after earthquakes using mobile phone data. PLoS one, 14(2), e0211375. https://doi.org/10.1371/journal.pone.0211375s

Zeigler, D. J., Brunn, S. D., \& Johnson, J. H., Jr. (1981). Evacuation from a nuclear technological disaster. Geographical Review, 71, 1-16. https://doi.org/10.2307/214548

Zhang, Y., Prater, C. S., \& Lindell, M. K. (2004). Risk area accuracy and evacuation from Hurricane Bret. Natural Hazards Review, 5(3), 115-120. https://doi.org/10.1061/(asce)1527$\underline{6988(2004) 5: 3(115)}$

Zhong, C., Manley, E., Arisona, S. M., Batty, M., \& Schmitt, G. (2015). Measuring variability of mobility patterns from multiday smart-card data. Journal of Computational Science, 9, 125130. https://doi.org/10.1016/j.jocs.2015.04.02 